KYUNGPOOK Math. J. 53(2013), 13-23

http://dx.doi.org/10.5666/KMJ.2013.53.1.13

\title{
Fekete-Szegö Problem for a Generalized Subclass of Analytic Functions
}

\author{
HALiT ORHAN* \\ Department of Mathematics, Faculty of Science, Ataturk University, 25240, Erzu- \\ rum, Turkey \\ e-mail : horhan@atauni.edu.tr \\ Nihat YagmuR \\ Department of Mathematics, Faculty of Science and Art, Erzincan University, \\ 24000, Erzincan, Turkey \\ e-mail : nhtyagmur@gmail.com \\ Murat Caglar \\ Department of Mathematics, Faculty of Science, Ataturk University, 25240, Erzu- \\ rum, Turkey \\ e-mail : mcaglar25@gmail.com
}

ABstract. In this present work, the authors obtain Fekete-Szegö inequality for certain normalized analytic function $f(z)$ defined on the open unit disk for which

$\frac{\lambda \beta z^{3}(L(a, c) f(z))^{\prime \prime \prime}+(2 \lambda \beta+\lambda-\beta) z^{2}(L(a, c) f(z))^{\prime \prime}+z(L(a, c) f(z))^{\prime}}{\lambda \beta z^{2}(L(a, c) f(z))^{\prime \prime}+(\lambda-\beta) z(L(a, c) f(z))^{\prime}+(1-\lambda+\beta)(L(a, c) f(z))} \quad(0 \leq \beta \leq \lambda \leq 1)$

lies in a region starlike with respect to 1 and is symmetric with respect to the real axis. Also certain applications of the main result for a class of functions defined by Hadamard product (or convolution) are given. As a special case of this result, Fekete-Szegö inequality for a class of functions defined through fractional derivatives are obtained.

\section{Introduction}

Let $\mathcal{A}$ denote the family of functions $f$ of the form

$$
f(z)=z+\sum_{k=2}^{\infty} a_{k} z^{k}
$$

which are analytic in the open unit disk $\mathcal{U}=\{z:|z|<1\}$. Further, let $\mathcal{S}$ denote the class of functions which are univalent in $\mathcal{U}$. For functions $f, g \in \mathcal{A}$, given by

* Corresponding Author.

Received March 16, 2011; accepted July 24, 2012.

2010 Mathematics Subject Classification: 30C45.

Key words and phrases: Fekete-Szegö problem, Analytic function, Coefficient inequality. 
$f(z)=z+\sum_{k=2}^{\infty} a_{k} z^{k}$ and $g(z)=z+\sum_{k=2}^{\infty} b_{k} z^{k}$, we define the Hadamard product (or convolution) of $f(z)$ and $g(z)$ by

$$
(f * g)(z):=z+\sum_{k=2}^{\infty} a_{k} b_{k} z^{k}=:(g * f)(z) \quad(z \in \mathcal{U}) .
$$

Note that $f * g \in \mathcal{A}$. If $f$ and $g$ are analytic in $\mathcal{U}$, we say that $f$ is subordinate to $g$, written symbolically as $f \prec g$ or $f(z) \prec g(z)(z \in \mathcal{U})$, if there exists a Schwarz function $w(z)$, which (by definition) is analytic in $\mathcal{U}$ with $w(0)=0$ and $|w(z)|<1$ in $\mathcal{U}$ such that $f(z)=g(w(z)), z \in \mathcal{U}$. In particular, if the function $g(z)$ is univalent in $\mathcal{U}$, then we have that $f(z) \prec g(z)(z \in \mathcal{U})$ if and only if $f(0)=g(0)$ and $f(\mathcal{U}) \subseteq g(\mathcal{U})$.

Let

$$
\varphi(a, c ; z):=z+\sum_{k=2}^{\infty} \frac{(a)_{k-1}}{(c)_{k-1}} z^{k} \quad(c \neq 0,-1,-2, \ldots ; z \in \mathcal{U})
$$

where $(\kappa)_{n}$ is the Pochhammer symbol (or the shifted factorial) in terms of the gamma function, given by

$$
(\kappa)_{n}:=\frac{\Gamma(\kappa+n)}{\Gamma(\kappa)}= \begin{cases}1 & n=0 \\ \kappa(\kappa+1)(\kappa+2) \ldots(\kappa+n-1) & n \in \mathbb{N}:=\{1,2, \ldots\} .\end{cases}
$$

Further, for $f \in \mathcal{A}$

$$
L(a, c) f(z)=\varphi(a, c ; z) * f(z)=z+\sum_{k=2}^{\infty} \frac{(a)_{k-1}}{(c)_{k-1}} a_{k} z^{k}
$$

where $L(a, c)$ is called Carlson-Shaffer operator [1] and the operator " * " stands for Hadamard product (or convolution product) of two power series as given by (1.2).

We notice that $L(a, a) f(z)=f(z), L(2,1) f(z)=z f^{\prime}(z), L(n+1,1) f(z)=$ $D^{n} f(z)$, where $D^{n} f(z)$ is the Ruscheweyh derivative of $f(z)$.

Let $\phi(z)$ be an analytic function with positive real part on $\mathcal{U}$ with $\phi(0)=1$, $\phi^{\prime}(0)>0$ which maps the unit disc $\mathcal{U}$ onto a region starlike with respect to 1 which is symmetric with to the real axis. Let $S^{*}(\phi)$ be the class of functions in $f \in \mathcal{S}$ for which

$$
\frac{z f^{\prime}(z)}{f(z)} \prec \phi(z) \quad(z \in \mathcal{U})
$$

and let $C(\phi)$ be the class of functions $f \in \mathcal{S}$ for which

$$
1+\frac{z f^{\prime \prime}(z)}{f^{\prime}(z)} \prec \phi(z) \quad(z \in \mathcal{U})
$$

where " $\prec$ " denotes the subordination between analytic functions. Above classes were defined and studied by Ma and Minda [4]. They have obtained the FeketeSzegö inequality for the functions in the class $C(\phi)$. Since $f \in C(\phi)$ if and only 
if $z f^{\prime} \in S^{*}(\phi)$, we get the Fekete-Szegö inequality for functions in the class $S^{*}(\phi)$. For a brief history of the Fekete-Szegö problem for the class of starlike, convex and close to convex functions, see the recent papers by Srivastava et. al. [11], Deniz and Orhan [2], Orhan and Güneş [5], Orhan et. al. [7, 8], Orhan and Raducanu [6].

Definition 1.1. Let $\phi(z)$ be a univalent starlike function with respect to 1 which maps the unit disk $\mathcal{U}$ onto a region in the half plane which is symmetric with respect to the real axis, $\phi(0)=1$, and $\phi^{\prime}(0)>0$. A function $f \in \mathcal{A}$ is in the class $P_{\lambda, \beta}^{a, c}(\phi)$, $0 \leq \beta \leq \lambda \leq 1$ if

$$
\frac{\lambda \beta z^{3}(L(a, c) f(z))^{\prime \prime \prime}+(2 \lambda \beta+\lambda-\beta) z^{2}(L(a, c) f(z))^{\prime \prime}+z(L(a, c) f(z))^{\prime}}{\lambda \beta z^{2}(L(a, c) f(z))^{\prime \prime}+(\lambda-\beta) z(L(a, c) f(z))^{\prime}+(1-\lambda+\beta)(L(a, c) f(z))} \prec \phi(z) .
$$

If we write $D_{\lambda, \beta} L(a, c) f(z)=\lambda \beta z^{2}(L(a, c) f(z))^{\prime \prime}+(\lambda-\beta) z(L(a, c) f(z))^{\prime}+(1-$ $\lambda+\beta)(L(a, c) f(z))$ then $f \in P_{\lambda, \beta}^{a, c}(\phi) \Leftrightarrow D_{\lambda, \beta} L(a, c) f \in S^{*}(\phi)$.

Also, we have

$$
D_{\lambda, \beta} L(a, c) f(z)=\Psi_{\lambda, \beta}^{a, c}(z) * f(z)
$$

where

$$
\Psi_{\lambda, \beta}^{a, c}(z)=z+\sum_{k=2}^{\infty}[1+(\lambda \beta k+\lambda-\beta)(k-1)] \frac{(a)_{k-1}}{(c)_{k-1}} z^{k}
$$

Lemma 1.1([4]). If $p_{1}=1+c_{1} z+c_{2} z^{2}+\ldots$ is analytic function with positive real part in $\mathcal{U}$, then

$$
\left|c_{2}-v c_{1}^{2}\right| \leqslant\left\{\begin{array}{lcr}
-4 v+2 & \text { if } & v \leqslant 0 \\
2 & \text { if } & 0 \leqslant v \leqslant 1 \\
4 v-2 & \text { if } & v \geqslant 1
\end{array}\right.
$$

When $v<0$ or $v>1$, the equality holds if and only if $p_{1}(z)$ is $(1+z) /(1-z)$ or one of its rotations. If $0<v<1$, then the equality holds if and only if $p_{1}(z)$ is $\left(1+z^{2}\right) /\left(1-z^{2}\right)$ or one of its rotations. If $v=0$, the equality holds if and only if

$$
p_{1}(z)=\left(\frac{1}{2}+\frac{1}{2} \gamma\right) \frac{1+z}{1-z}+\left(\frac{1}{2}-\frac{1}{2} \gamma\right) \frac{1-z}{1+z} \quad(0 \leqslant \gamma \leqslant 1),
$$

or one of its rotations. If $v=1$, the equality holds if and only if $p_{1}(z)$ is the reciprocal of one of the functions such that the equality holds in the case of $v=0$. The above upper bound is sharp. When $0<v<1$, it can be improved as follows:

$$
\left|c_{2}-v c_{1}^{2}\right|+v\left|c_{1}\right|^{2} \leqslant 2 \quad\left(0<v \leqslant \frac{1}{2}\right)
$$


and

$$
\left|c_{2}-v c_{1}^{2}\right|+(1-v)\left|c_{1}\right|^{2} \leqslant 2 \quad\left(\frac{1}{2}<v \leqslant 1\right) .
$$

\section{Fekete-Szegö Problem}

In this section, we will give some upper bounds for the Fekete-Szegö functional $\left|a_{3}-\mu a_{2}^{2}\right|$. In order to prove our main results we have to recall the following. Firstly, the following information will be used in the proof of the Theorem 2.1. By geometric interpretation there exists a function $w$ satisfying the conditions of the Schwarz lemma such that

$$
\frac{z\left(D_{\lambda, \beta} L(a, c) f(z)\right)^{\prime}}{D_{\lambda, \beta} L(a, c) f(z)}=\phi(w(z)) \quad(z \in \mathcal{U}) .
$$

Secondly, we introduce the following functions which will be used in the discussion of sharpness of our results.

Corresponding to the function $\Psi_{\lambda, \beta}^{a, c}(z)$ defined by (1.8), we also consider the function $\Psi_{\lambda, \beta}^{a, c}(z)^{(-1)}$ given by

$$
\Psi_{\lambda, \beta}^{a, c}(z)^{(-1)}=z+\sum_{k=2}^{\infty} \frac{(c)_{k-1}}{(a)_{k-1}[1+(\lambda \beta k+\lambda-\beta)(k-1)]} z^{k}
$$

where inverse is taken with respect to Hadamard product.

Using (1.7), (2.1) and logarithmic differentiation it can be deduce that $f \in$ $P_{\lambda, \beta}^{a, c}(\phi)$ if and only if

$$
f(z)=\Psi_{\lambda, \beta}^{a, c}(z)^{(-1)} *\left\{z \exp \left(\int_{0}^{z} \frac{\phi(w(t))-1}{t} d t\right)\right\}
$$

for some function $w(z)$ satisfying the conditions of the Schwarz Lemma.

Define the function $G$ in $\mathcal{U}$ by

$$
G(z)=\frac{1}{z}\left[\Psi_{\lambda, \beta}^{a, c}(z)^{(-1)} *\left\{z \exp \left(\int_{0}^{z} \frac{\phi(\xi)-1}{\xi} d \xi\right)\right\}\right] .
$$

Also we consider the following extremal function

$$
K(z, \theta, \tau)=\Psi_{\lambda, \beta}^{a, c}(z)^{(-1)} * z \exp \left(\int_{0}^{z}\left[\phi\left(\frac{e^{i \theta} \xi(\xi+\tau)}{1+\tau \xi}\right)-1\right] \frac{d \xi}{\xi}\right) \quad(0 \leqslant \theta \leqslant 2 \pi, 0 \leqslant \tau \leqslant 1) .
$$

Note that $K(z, 0,1)=z G(z)$ defined by $(2.3)$ and $K(z, \theta, 0)$ is an odd function. 
Theorem 2.1. Let $\phi(z)=1+B_{1} z+B_{2} z^{2}+\ldots$. If $f(z)$ given by (1.1) belongs to the class $P_{\lambda, \beta}^{a, c}(\phi)$, then

$$
\left|a_{3}-\mu a_{2}^{2}\right| \leqslant\left\{\begin{array}{lc}
\frac{c(c+1) B_{2}}{2 a(a+1) A_{2}}-\frac{\mu c^{2} B_{1}^{2}}{a^{2} A_{1}^{2}}+\frac{c(c+1) B_{1}^{2}}{2 a(a+1) A_{2}} & \text { if } \quad \mu \leqslant \sigma_{1}, \\
\frac{c(c+1) B_{1}}{2 a(a+1) A_{2}} & \text { if } \sigma_{1} \leqslant \mu \leqslant \sigma_{2}, \\
-\frac{c(c+1) B_{2}}{2 a(a+1) A_{2}}+\frac{\mu c^{2} B_{1}^{2}}{a^{2} A_{1}^{2}}-\frac{c(c+1) B_{1}^{2}}{2 a(a+1) A_{2}} & \text { if } \quad \mu \geqslant \sigma_{2},
\end{array}\right.
$$

where

$$
\begin{aligned}
& \sigma_{1}:=\frac{a(c+1) A_{1}^{2}\left\{\left(B_{2}-B_{1}\right)+B_{1}^{2}\right\}}{2 c(a+1) A_{2} B_{1}^{2}}, \\
& \sigma_{2}:=\frac{a(c+1) A_{1}^{2}\left\{\left(B_{2}+B_{1}\right)+B_{1}^{2}\right\}}{2 c(a+1) A_{2} B_{1}^{2}}
\end{aligned}
$$

and

$$
A_{1}=(2 \lambda \beta+\lambda-\beta+1), \quad A_{2}=(6 \lambda \beta+2 \lambda-2 \beta+1) \quad(0 \leq \beta \leq \lambda \leq 1) .
$$

Each of the estimates in (2.5) is sharp for the function $K(z, \theta, \tau)$ given by (2.4).

Proof. For $f(z) \in P_{\lambda, \beta}^{a, c}(\phi)$, let

(2.6) $p(z)$

$$
\begin{aligned}
& =\frac{\lambda \beta z^{3}(L(a, c) f(z))^{\prime \prime \prime}+(2 \lambda \beta+\lambda-\beta) z^{2}(L(a, c) f(z))^{\prime \prime}+z(L(a, c) f(z))^{\prime}}{\lambda \beta z^{2}(L(a, c) f(z))^{\prime \prime}+(\lambda-\beta) z(L(a, c) f(z))^{\prime}+(1-\lambda+\beta)(L(a, c) f(z))} \\
& =1+b_{1} z+b_{2} z^{2}+\ldots
\end{aligned}
$$

From (2.6), we obtain

$$
\frac{a}{c} A_{1} a_{2}=b_{1} \text { and } \frac{2 a(a+1)}{c(c+1)} A_{2} a_{3}=A_{1}^{2} \frac{a^{2}}{c^{2}} a_{2}^{2}+b_{2} .
$$

Since $\phi(z)$ is univalent and $p \prec \phi$, the function

$$
p_{1}(z)=\frac{1+\phi^{-1}(p(z))}{1-\phi^{-1}(p(z))}=1+c_{1} z+c_{2} z^{2}+\ldots
$$

is analytic and has positive real part in $\mathcal{U}$. We also have

$$
p(z)=\phi\left(\frac{p_{1}(z)-1}{p_{1}(z)+1}\right)
$$

and thus, we get

$$
b_{1}=\frac{1}{2} B_{1} c_{1} \text { and } b_{2}=\frac{1}{2} B_{1}\left(c_{2}-\frac{1}{2} c_{1}^{2}\right)+\frac{1}{4} B_{2} c_{1}^{2} .
$$


Hence, we have

$$
a_{3}-\mu a_{2}^{2}=\frac{c(c+1) B_{1}}{4 a(a+1) A_{2}}\left\{c_{2}-v c_{1}^{2}\right\}
$$

where

$$
v:=\frac{1}{2}\left(1-\frac{B_{2}}{B_{1}}+\frac{2 a c(a+1) A_{2} \mu-a^{2}(c+1) A_{1}^{2}}{a^{2}(c+1) A_{1}^{2}} B_{1}\right) .
$$

If $\mu \leqslant \sigma_{1}$, then, according to Lemma 1.1, we get

$$
\begin{aligned}
& \left|a_{3}-\mu a_{2}^{2}\right| \\
= & \frac{c(c+1) B_{1}}{4 a(a+1) A_{2}}\left|c_{2}-c_{1}^{2}\left[\frac{1}{2}\left(1-\frac{B_{2}}{B_{1}}+\frac{2 a c(a+1) A_{2} \mu-a^{2}(c+1) A_{1}^{2}}{a^{2}(c+1) A_{1}^{2}} B_{1}\right)\right]\right|
\end{aligned}
$$

and thus,

$$
\left|a_{3}-\mu a_{2}^{2}\right| \leqslant \frac{c(c+1) B_{2}}{2 a(a+1) A_{2}}-\frac{\mu c^{2} B_{1}^{2}}{a^{2} A_{1}^{2}}+\frac{c(c+1) B_{1}^{2}}{2 a(a+1) A_{2}},
$$

which is the first assertion of (2.5).

Next, if $\mu \geqslant \sigma_{2}$, by applying Lemma 1.1, we get

$$
\left|a_{3}-\mu a_{2}^{2}\right| \leqslant-\frac{c(c+1) B_{2}}{2 a(a+1) A_{2}}+\frac{\mu c^{2} B_{1}^{2}}{a^{2} A_{1}^{2}}-\frac{c(c+1) B_{1}^{2}}{2 a(a+1) A_{2}}
$$

which is the third assertion of (2.5).

If $\sigma_{1} \leqslant \mu \leqslant \sigma_{2}$, by using again Lemma 1.1, we obtain

$$
\left|a_{3}-\mu a_{2}^{2}\right| \leqslant \frac{c(c+1) B_{1}}{2 a(a+1) A_{2}}
$$

which is the second part of the assertion (2.5).

We now obtain sharpness of the estimates in (2.5). If $\mu<\sigma_{1}$ or $\mu>\sigma_{2}$, then equality holds in (2.5) if and only if equality holds in (2.8). This happens if and only if $c_{1}=2$ and $c_{2}=2$. Thus $w(z)=z$. It follows that the extremal function is of the form $K(z, 0,1)$ defined by $(2.4)$ or one of its rotations.

If $\mu=\sigma_{2}$, the equality holds if and only if $\left|c_{2}\right|=2$. In this case, we have

$$
\frac{1}{2}\left(1-\frac{B_{2}}{B_{1}}+\frac{2 a c(a+1) A_{2} \mu-a^{2}(c+1) A_{1}^{2}}{a^{2}(c+1) A_{1}^{2}} B_{1}\right)=0 .
$$

Therefore the extremal function $f$ is $K(z, \theta, \tau)$ or one of its rotations.

Similarly, $\mu=\sigma_{1}$ is equivalent to

$$
p_{1}(z)=\frac{1+\tau}{2}\left(\frac{1+z}{1-z}\right)+\frac{1-\tau}{2}\left(\frac{1-z}{1+z}\right) \quad(0<\tau<1 ; z \in \mathcal{U})
$$

Thus the extremal function is $K(z, 0, \tau)$ or one of its rotations. 
Finally if $\sigma_{1} \leqslant \mu \leqslant \sigma_{2}$, then equality holds if $\left|c_{1}\right|=0$ and $\left|c_{2}\right|=2$. Equivalently, we have

$$
h(z)=\frac{1+\tau z^{2}}{1-\tau z^{2}} \quad(0 \leqslant \tau \leqslant 1 ; z \in \mathcal{U}) .
$$

Therefore the extremal function $f$ is $K(z, 0,0)$ or one of its rotations. The proof of Theorem 2.1 is now completed.

Remark 2.2. If $\sigma_{1} \leqslant \mu \leqslant \sigma_{2}$, then, in view of Lemma 1.1, Theorem 2.1 can be improved. Let $\sigma_{3}$ given by

$$
\sigma_{3}:=\frac{a(c+1) A_{1}^{2}\left\{B_{1}^{2}+B_{2}\right\}}{2 c(a+1) A_{2} B_{1}^{2}} .
$$

If $\sigma_{1} \leqslant \mu \leqslant \sigma_{3}$, then

$$
\begin{aligned}
& \left|a_{3}-\mu a_{2}^{2}\right|+\frac{a(c+1) A_{1}^{2}}{2 c(a+1) A_{2} B_{1}^{2}}\left[B_{1}-B_{2}+\frac{2 a c(a+1) A_{2} \mu-a^{2}(c+1) A_{1}^{2}}{a^{2}(c+1) A_{1}^{2}} B_{1}^{2}\right]\left|a_{2}\right|^{2} \\
& \leqslant \frac{c(c+1) B_{1}}{2 a(a+1) A_{2}} .
\end{aligned}
$$

If $\sigma_{3} \leqslant \mu \leqslant \sigma_{2}$, then

$$
\begin{aligned}
& \left|a_{3}-\mu a_{2}^{2}\right|+\frac{a(c+1) A_{1}^{2}}{2 c(a+1) A_{2} B_{1}^{2}}\left[B_{1}+B_{2}-\frac{2 a c(a+1) A_{2} \mu-a^{2}(c+1) A_{1}^{2}}{a^{2}(c+1) A_{1}^{2}} B_{1}^{2}\right]\left|a_{2}\right|^{2} \\
& \leqslant \frac{c(c+1) B_{1}}{2 a(a+1) A_{2}}
\end{aligned}
$$

where $A_{1}$ and $A_{2}$ are given by Theorem 2.1.

Proof. If $\sigma_{1} \leqslant \mu \leqslant \sigma_{3}$, we have

$$
\begin{aligned}
& \left|a_{3}-\mu a_{2}^{2}\right|+\left(\mu-\sigma_{1}\right)\left|a_{2}\right|^{2} \\
= & \frac{c(c+1) B_{1}}{4 a(a+1) A_{2}}\left|c_{2}-v c_{1}^{2}\right|+\left(\mu-\sigma_{1}\right) \frac{c^{2} B_{1}^{2}}{4 a^{2} A_{1}^{2}}\left|c_{1}\right|^{2} \\
= & \frac{c(c+1) B_{1}}{4 a(a+1) A_{2}}\left|c_{2}-v c_{1}^{2}\right|+\left(\mu-\frac{a(c+1) A_{1}^{2}\left\{\left(B_{2}-B_{1}\right)+B_{1}^{2}\right\}}{2 c(a+1) A_{2} B_{1}^{2}}\right) \frac{c^{2} B_{1}^{2}}{4 a^{2} A_{1}^{2}}\left|c_{1}\right|^{2} \\
= & \frac{c(c+1) B_{1}}{2 a(a+1) A_{2}}\left[\frac{1}{2}\left|c_{2}-v c_{1}^{2}\right|\right. \\
& \left.+\frac{1}{2}\left(\frac{2 \mu c(a+1) A_{2} B_{1}^{2}-a(c+1) A_{1}^{2}\left[B_{1}^{2}-B_{1}+B_{2}\right]}{2 a(c+1) A_{2}^{2} B_{1}}\right)\left|c_{1}\right|^{2}\right] \\
= & \frac{c(c+1) B_{1}}{2 a(a+1) A_{2}}\left\{\frac{1}{2}\left[\left|c_{2}-v c_{1}^{2}\right|+v\left|c_{1}\right|^{2}\right]\right\} \leqslant \frac{c(c+1) B_{1}}{2 a(a+1) A_{2}} .
\end{aligned}
$$


Similarly, if $\sigma_{3} \leqslant \mu \leqslant \sigma_{2}$, we can write the following

$$
\begin{aligned}
& \left|a_{3}-\mu a_{2}^{2}\right|+\left(\sigma_{2}-\mu\right)\left|a_{2}\right|^{2} \\
= & \frac{c(c+1) B_{1}}{4 a(a+1) A_{2}}\left|c_{2}-v c_{1}^{2}\right|+\left(\sigma_{2}-\mu\right) \frac{c^{2} B_{1}^{2}}{4 a^{2} A_{1}^{2}}\left|c_{1}\right|^{2} \\
= & \frac{c(c+1) B_{1}}{4 a(a+1) A_{2}}\left|c_{2}-v c_{1}^{2}\right|+\left(\frac{a(c+1) A_{1}^{2}\left[B_{1}^{2}+B_{1}+B_{2}\right]}{2 c(a+1) A_{2} B_{1}^{2}}-\mu\right) \frac{c^{2} B_{1}^{2}}{4 a^{2} A_{1}^{2}}\left|c_{1}\right|^{2} \\
= & \frac{c(c+1) B_{1}}{2 a(a+1) A_{2}}\left[\frac{1}{2}\left|c_{2}-v c_{1}^{2}\right|\right. \\
& \left.+\frac{1}{2}\left(\frac{a(c+1) A_{1}^{2}\left[B_{1}^{2}+B_{1}+B_{2}\right]-2 \mu c(a+1) A_{2} B_{1}^{2}}{2 a(c+1) A_{2}^{2} B_{1}}\right)\left|c_{1}\right|^{2}\right] \\
= & \frac{c(c+1) B_{1}}{2 a(a+1) A_{2}}\left\{\frac{1}{2}\left[\left|c_{2}-v c_{1}^{2}\right|+(1-v)\left|c_{1}\right|^{2}\right]\right\} \leqslant \frac{c(c+1) B_{1}}{2 a(a+1) A_{2}} .
\end{aligned}
$$

Thus, the proof of Remark 2.2 is completed.

\section{Applications to functions defined by fractional derivatives}

For fixed $g \in A$, let $P_{\lambda, \beta}^{a, c, g}(\phi)$ be class of functions $f \in A$ for which $(f * g) \in$ $P_{\lambda, \beta}^{a, c}(\phi)$. In order to introduce the class $P_{\lambda, \beta}^{a, c, g}(\phi)$, we need the following:

Definition 3.1([9]). Let $f(z)$ be analytic in a simply connected region of the $z$-plane containing the origin. The fractional derivative of $f$ of order $\gamma$ is defined by

$$
D_{z}^{\gamma} f(z)=\frac{1}{\Gamma(1-\gamma)} \frac{d}{d z} \int_{0}^{z} \frac{f(\zeta)}{(z-\zeta)^{\gamma}} d \zeta \quad(0 \leqslant \gamma<1)
$$

where the multiplicity of $(z-\zeta)^{\gamma}$ is removed by requiring that $\log (z-\zeta)$ is real for $z-\zeta>0$.

Using the above Definition 3.1 and its known extensions involving fractional derivatives and fractional integrals, Owa and Srivastava [9] introduced the operator $\Omega^{\gamma}: A \rightarrow A$ defined by

$$
\Omega^{\gamma} f(z)=\Gamma(2-\gamma) z^{\gamma} D_{z}^{\gamma} f(z) \quad(\gamma \neq 2,3,4, \ldots) .
$$

The class $P_{\lambda, \beta}^{a, c, \gamma}(\phi)$ consists of functions $f \in A$ for which $\Omega^{\gamma} f \in P_{\lambda, \beta}^{a, c}(\phi)$. Note that $P_{0,0}^{a, a}(\phi) \equiv S^{*}(\phi)$ and $P_{\lambda, \beta}^{a, c, \gamma}(\phi)$ is the special case of the class $P_{\lambda, \beta}^{a, c, g}(\phi)$ when

$$
g(z)=z+\sum_{k=2}^{\infty} \frac{\Gamma(k+1) \Gamma(2-\gamma)}{\Gamma(k+1-\gamma)} z^{k}
$$

Let

$$
g(z)=z+\sum_{k=2}^{\infty} g_{k} z^{k} \quad\left(g_{k}>0\right)
$$


Since $L(a, c) f(z) \in P_{\lambda, \beta}^{a, c, g}(\phi)$ if and only if $L(a, c) f(z) * g(z) \in P_{\lambda, \beta}^{a, c}(\phi)$, we obtain the coefficient estimate for functions in the class $P_{\lambda, \beta}^{a, c, g}(\phi)$, from the corresponding estimate for functions in the class $P_{\lambda, \beta}^{a, c}(\phi)$.

Applying Theorem 2.1 for the function

$$
\begin{aligned}
L(a, c) f(z) * g(z) & =z+\sum_{k=2}^{\infty} \frac{(a)_{k-1}}{(c)_{k-1}} a_{k} g_{k} z^{k} \\
& =z+\frac{a}{c} a_{2} g_{2} z^{2}+\ldots
\end{aligned}
$$

We get the following Theorem 3.1 after an obvious change of the parameter $\mu$ :

Theorem 3.1. Let $g(z)=z+\sum_{k=2}^{\infty} g_{k} z^{k},\left(g_{k}>0\right)$, and let the function $\phi(z)$ be given by $\phi(z)=1+\sum_{n=1}^{\infty} B_{n} z^{n}$. If $L(a, c) f(z)$ defined by (1.5) belongs to the class $P_{\lambda, \beta}^{a, c, g}(\phi)$, then

$$
\begin{aligned}
& \left|a_{3}-\mu a_{2}^{2}\right|
\end{aligned}
$$

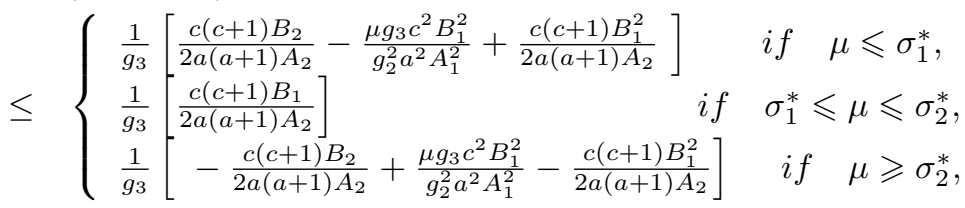

where

$$
\begin{aligned}
\sigma_{1}^{*}: & =\frac{g_{2}^{2} a(c+1) A_{1}^{2}\left\{\left(B_{2}-B_{1}\right)+B_{1}^{2}\right\}}{2 g_{3} c(a+1) A_{2} B_{1}^{2}}, \\
\sigma_{2}^{*}: & =\frac{g_{2}^{2} a(c+1) A_{1}^{2}\left\{\left(B_{2}+B_{1}\right)+B_{1}^{2}\right\}}{2 g_{3} c(a+1) A_{2} B_{1}^{2}} .
\end{aligned}
$$

The result is sharp.

Since

$$
\left(\Omega^{\gamma} L(a, c) f\right)(z)=z+\sum_{k=2}^{\infty} \frac{\Gamma(k+1) \Gamma(2-\gamma)(a)_{k-1}}{\Gamma(k+1-\gamma)(c)_{k-1}} a_{k} z^{k}
$$

we have

$$
g_{2}:=\frac{\Gamma(3) \Gamma(2-\gamma)}{\Gamma(3-\gamma)}=\frac{2}{2-\gamma}
$$

and

$$
g_{3}:=\frac{\Gamma(4) \Gamma(2-\gamma)}{\Gamma(4-\gamma)}=\frac{6}{(2-\gamma)(3-\gamma)} .
$$

For $g_{2}$ and $g_{3}$ given by above equalities, Theorem 3.1 reduces to the following: 
Theorem 3.2. Let $g(z)=z+\sum_{k=2}^{\infty} g_{k} z^{k},\left(g_{k}>0\right)$ and let the function $\phi(z)$ be given by $\phi(z)=1+\sum_{n=1}^{\infty} B_{n} z^{n}$. If $L(a, c) f(z)$ defined by (1.5) belongs to the class $P_{\lambda, \beta}^{a, c, g}(\phi)$, then

$$
\begin{aligned}
& \left|a_{3}-\mu a_{2}^{2}\right| \\
& \leqslant\left\{\begin{array}{l}
\frac{(2-\gamma)(3-\gamma)}{6}\left[\frac{c(c+1) B_{2}}{2 a(a+1) A_{2}}-\frac{3(2-\gamma) \mu c^{2} B_{1}^{2}}{2(3-\gamma) a^{2} A_{1}^{1}}+\frac{c(c+1) B_{1}^{2}}{2 a(a+1) A_{2}}\right] \quad \text { if } \quad \eta \leqslant \sigma_{1}^{* *}, \\
\frac{(2-\gamma)(3-\gamma)}{6}\left[\frac{c(c+1) B_{1}}{2 a(a+1) A_{2}}\right] \quad \text { if } \sigma_{1}^{* *} \leqslant \eta \leqslant \sigma_{2}^{* *}, \\
\frac{(2-\gamma)(3-\gamma)}{6}\left[-\frac{c(c+1) B_{2}}{2 a(a+1) A_{2}}+\frac{3(2-\gamma) \mu c^{2} B_{1}^{2}}{2(3-\gamma) a^{2} A_{1}^{2}}-\frac{c(c+1) B_{1}^{2}}{2 a(a+1) A_{2}}\right] \quad \text { if } \eta \geqslant \sigma_{2}^{* *},
\end{array}\right.
\end{aligned}
$$

where

$$
\begin{aligned}
\sigma_{1}^{* *} & :=\frac{(3-\gamma) a(c+1) A_{1}^{2}\left\{\left(B_{2}-B_{1}\right)+B_{1}^{2}\right\}}{3(2-\gamma) c(a+1) A_{2} B_{1}^{2}}, \\
\sigma_{2}^{* *}: & =\frac{(3-\gamma) a(c+1) A_{1}^{2}\left\{\left(B_{2}+B_{1}\right)+B_{1}^{2}\right\}}{3(2-\gamma) c(a+1) A_{2} B_{1}^{2}} .
\end{aligned}
$$

The result is sharp.

Remark 3.3. When $a=c, \quad \lambda=\beta=0, \quad B_{1}=8 / \pi^{2}$ and $B_{2}=16 / 3 \pi^{2}$, Theorem 3.2 reduces to a result of Srivastava and Mishra ( [11], Theorem 8, p. 64 ) for a class of which $\Omega^{\gamma} f(z)$ is a parabolic starlike function (see [3], [10]).

Acknowledgements The authors thank the referees for their valuable suggestions to improve the paper.

The present investigation was supported by Ataturk University Rectorship under "The Scientific and Research Project of Ataturk University" , Project No: $2012 / 173$.

\section{References}

[1] B. C. Carlson and D. B. Shaffer, Starlike and prestarlike hypergeometric functions, SIAM J. Math. Anal., 15(1984), 737-745.

[2] E. Deniz and H. Orhan, The Fekete-Szegö Problem for A Generalized Subclass of Analytic Functions, Kyungpook Math. J., 50(2010), 37-47.

[3] A. W. Goodman, Uniformly convex functions, Ann. Polon. Math., 56(1991), 87-92.

[4] W. Ma and D. Minda, A unified treatment of some special classes of univalent functions, in Proceeding of the conference on complex analysis, Z. Li, F. Ren, L. Yang and S. Zhang (Eds.), Int. Press, (1994), 157-169. 
[5] H. Orhan and E. Güneş, Fekete-Szegö Inequality for Certain Subclass of Analytic Functions, General Math., 14(1)(2005), 41-54.

[6] H. Orhan and D. Răducanu, Fekete-Szegö problem for strongly starlike functions associated with generalized hypergeometric functions, Math. Comput. Modelling, 50(2009), 430-438.

[7] H. Orhan, E. Deniz and D. Răducanu, The Fekete-Szegö problem for subclasses of analytic functions defined by a differential operator related to conic domains, Comput. Math. Appl., 59(2010), 283-295.

[8] H. Orhan, N. Yağmur and E. Deniz, Coefficient Inequality For A Generalized Subclass Of Analytic Functions, Bulletin of the Trans. Univ. of Brasov, Vol 4(53), No. 1 - 2011 Series III: Mathematics, Informatics, Physics, 51-58.

[9] S. Owa and H. M. Srivastava, Univalent and starlike functions generalized by hypergeometric functions, Canad. J. Math., 39(1987), 1057-1077.

[10] F. Ronning, Uniformly convex functions and a corresponding class of starlike functions, Proc. Amer. Math. Soc., 118(1993), 189-196.

[11] H. M. Srivastava, A. K. Mishra and M. K. Das, The Fekete-Szegö problem for a subclass of close-to-convex functions, Complex variables, Theory Appl., 44(2001), 145-163. 